\title{
Tourette Syndrome and Klippel-Feil Anomaly in a Child with Chromosome 22q11 Duplication
}

\author{
Raymond A. Clarke, ${ }^{1}$ Zhi Ming Fang, ${ }^{1}$ Ashish D. Diwan, ${ }^{1}$ and Donald L. Gilbert ${ }^{2}$ \\ ${ }^{1}$ St George Clinical School, Faculty of Medicine, St George Hospital, University of NSW, Kogarah, NSW 2217, Australia \\ ${ }^{2}$ Movement Disorders Clinics, Division of Pediatric Neurology, Cincinnati Children's Hospital Medical Center, \\ ML \# 11006 - Neurology, 3333 Burnet Avenue, Cincinnati, OH 45229-3039, USA
}

Correspondence should be addressed to Donald L. Gilbert, donald.gilbert@cchmc.org

Received 14 July 2009; Accepted 26 October 2009

Recommended by Jean-Pierre Fryns

This is the first case description of the association of Klippel-Feil Syndrome (KFS), Tourette Syndrome (TS), Motor Stereotypies, and Obsessive Compulsive Behavior, with chromosome 22q11.2 Duplication Syndrome (22q11DupS). Neuropsychiatric symptoms in persons with 22q11.2 deletion, including obsessive compulsiveness, anxiety, hyperactivity, and one prior case report of TS, have been attributed to low copy number effects on Catechol-O-Methyltransferase (COMT). However, the present unique case of 22q11DupS and TS suggests a more complex relationship involving another gene(s) at or near this locus.

Copyright ( $) 2009$ Raymond A. Clarke et al. This is an open access article distributed under the Creative Commons Attribution License, which permits unrestricted use, distribution, and reproduction in any medium, provided the original work is properly cited.

\section{Introduction}

Klippel-Feil Syndrome (KFS) involves a congenital fusion of vertebrae in the cervical spine [1]. Tourette Syndrome (TS) is a heritable neuropsychiatric disorder characterized by persistent, childhood-onset tics that fluctuate in intensity, migrate in anatomic location, and are performed in response to sensory urges [2]. Motor stereotypies usually have younger onset, are much more stable phenomenologically, and occur involuntarily with excitement or other stimulation [3]. Longstanding clinical observations that high doses of amphetamines can induce stereotypies and tics [4], while dopamine receptor blocking agents inhibit them [5], support a hyperdopaminergic neurotransmission or receptor hypersensitivity model for these symptoms, although the dopamine/tic relationship is likely much more complex [6].

Large genetic association and linkage studies to date have failed to implicate genes for dopamine synthesis or metabolism as causes of TS, but clinical presentations of rare genetic diseases involving dopamine pathways are supportive, as in a recently reported case of TS in a girl with chromosome 22q11 deletion [7]. This region contains Catechol-O-Methyltransferase (COMT), which degrades dopamine, norepinephrine, and epinephrine. Low-COMT gene copy number has been associated with obsessive compulsive and hyperactive psychopathology in velocardiofacial syndrome [8], and lesser-COMT activity theoretically could increase catecholamines and hyperkinetic movements.

We report clinical and genetic characterization of the first case of 22q11DupS and TS, KFS, and stereotypies, suggesting that both low and high copy numbers of 22q11 may generate hyperkinetic and obsessive compulsive symptoms.

\section{Case Presentation}

The patient, a Caucasian male diagnosed with KFS, was referred at age of 9 years for evaluation of repetitive movements. He presented with motor stereotypy in the 1st year of life which persists to the present: a patterned movement involving the flapping of both hands, and sometimes body and leg stiffening is occurring daily, with excitement. From age 6 to his present age, 12 years, he has had a series of mild tics including eye blinking, nose twitching, leg and toe pointing, and repetitive coughing and sniffing, consistent with a diagnosis of TS. He also has obsessive compulsive behaviour (OCB) and anxiety, with excessive rumination, 


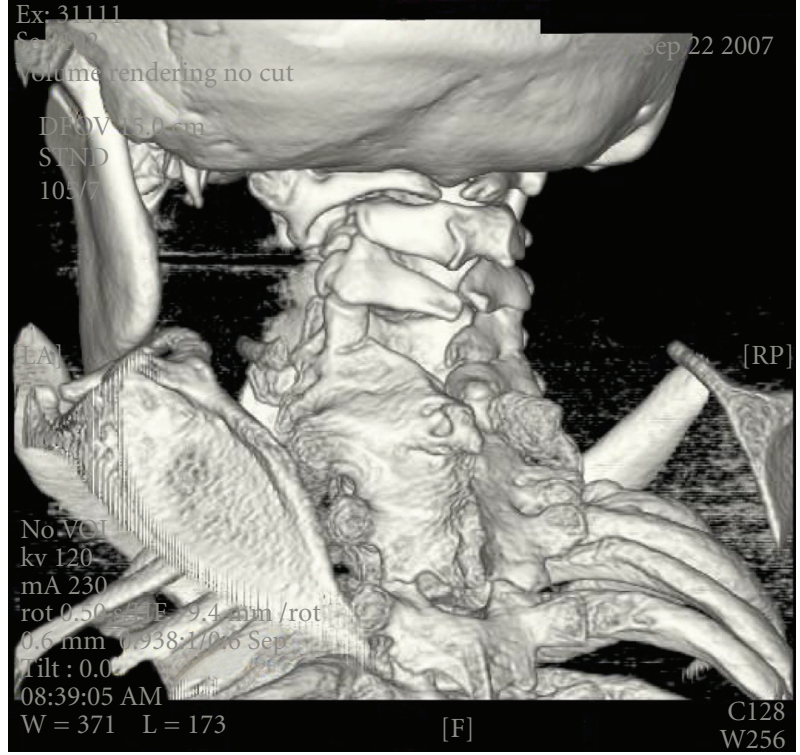

Figure 1: Computed Tomography of the C Spine, with 3D reconstruction, showing Klippel-Feil anomaly comprising of the fusion of multiple cervical and upper thoracic vertebrae.

but no Attention Deficit Hyperactivity Disorder (ADHD). He has seen a psychologist for dysthymia and adjustment but takes no neurologic or psychiatric medications. At school, academic performance is average to above. Past Medical History includes full-term birth, at $3.1 \mathrm{~kg}$. Development includes walking by 16 months and speaking normally by 2 years. Surgical history includes a Woodward Procedure for Sprengel's Deformity of the scapula and Cervical Spine Decompression and Stabilization. Review of systems includes parasomnias and migraines. Family history is negative for tics, OCB, or other neurologic or psychiatric disorders.

General examination revealed a barrel-chested appearance, a short neck with low posterior hairline, abnormal scapulae, and webbing of toes. At age of 12 years, weight was $36.6 \mathrm{~kg}$ (75\%ile), height was $126.4 \mathrm{~cm}(<5 \%$ ile), body mass index was 34 (>95\%ile), and head circumference was $50.5 \mathrm{~cm}$. Palate and cardiac exams were normal. Neurologic exam was notable for normal language and gregarious social interactions. Occasional tics and the stereotypy were observed. Cranial nerve exam was normal. Distal strength was diminished in both hands and thumb abductors. Reflexes were $2+$ and symmetric. Fine movements of the hands were clumsy and slow. Sensation was diminished to light touch in the hands. Current clinical ratings showed Yale Global Tic Severity Scale (YGTSS) total tic score of 6 (mild), Child Yale-Brown Obsessive Compulsive Scale (CY-BOCS) score of 16 (mild/moderate), and the DuPaul ADHD Rating Scale (ADHDRS) score of 17 (subthreshold).

Imaging studies include CT scans that revealed fusion of cervical and thoracic vertebrae (Figure 1), scoliosis, hypoplastic thumbs, and several digits of the feet and hands.

Due to the complex phenotype, we evaluated the patient's DNA using comparative genomic hybridization (CGH) analysis (BeadChip technology with a Single Nucleotide
Polymorphism based array; Illumina HD Human610-quad BeadChip platform). This revealed a $\sim 3 \mathrm{Mb}$ hemizygous microduplication of chromosome $22 \mathrm{q} 11.2$, the same region as the classical 22q11 microdeletion syndrome [9], as well as the 22q11.2 duplication with a less well-characterized phenotype [10]. This region contains genes coding for approximately 20 proteins expressed in brain and/or bone.

Followup negative testing of the parents demonstrated that this duplication was de novo.

To further characterize this boy's COMT genotype, we generated a lymphocyte cell line. DNA nucleotide sequence analysis indicated that the patient was heterozygous for the G675A COMT haplotype (Figure 2(a)i) and that the chromatogram peak height for the $675 \underline{G}$ variant was consistently two times higher than that of the $675 \underline{A}$ variant (Figure 2(a)i). This suggests that the 675G (higherexpressing) allele [11] was duplicated. We then compared $\mathrm{mRNA} / \mathrm{cDNA}$ sequence for the patient and a normal individual. The $\mathrm{G}: \underline{A}$ ratio for the normal control heterozygote was slightly $>1$ (Figure 2(a)iii) consistent with the instability of the $675 \underline{A}$ mRNA transcript [11]. In contrast, the $\underline{G}$ : $\underline{A}$ ratio for the patient was $>2$ (Figure 2(a)ii) which again suggested that the patient has a higher than normal level of expression of the COMT gene.

To directly compare COMT gene expression levels, we optimized a proven two-step, high-temperature, semiquantitative RTPCR protocol (denature at $94^{\circ} \mathrm{C}$ for 30 seconds followed by extension at $71^{\circ} \mathrm{C}$ for 40 seconds for 33 cycles) [12] using primers specific for either the $675 \mathrm{G}$ or $675 \mathrm{~A}$ : the forward $675 \mathrm{G}$ primer (COMTValF $5^{\prime}$-ATGGTGGATTTCGCTGGCGT-3') and the 675 primer (COMT-MetF 5'-ATGGTGGATTTCGCTGGCAT$3^{\prime}$ ) were specific for sequence from exon 4 and the reverse primer (COMT-R 5'-CTTCCGCAGCAGGCCACATT-3') for sequence from exon 5. Only the patient (Figure 2(b), lane 2) expressed the $675 \mathrm{~A}$ allele and both the patient and two normal controls (lanes 3 and 4) appeared to have comparable levels of expression of the $675 \underline{G}$ allele, as evident from the equivalent intensity of amplicons.

To assess possible clinical implications of this finding, cerebrospinal fluid was obtained via lumbar puncture for assessment of neurotransmitter levels. Routine cells ( 1 white, 1 red blood cell), protein, and glucose were normal. Levels of CSF neurotransmitter metabolites were obtained through HPLC testing commercially (Medical Neurogenetics, Atlanta, Georgia). The level of 3-O-methyldopa, a metabolite of $\mathrm{L}$ DOPA via COMT, was normal at $19 \mathrm{nmol} / \mathrm{L}$ (normal $<100$ ). The level of Homovanillic acid, a final common pathway metabolite of dopamine and norepinephrine via COMT, was normal at $279 \mathrm{nmol} / \mathrm{L}(167-563)$.

\section{Discussion}

We describe a boy with KFS, TS, stereotypy, and obsessive compulsive symptoms in association with de novo 22q11DupS with normal parental genotype and phenotype. This is the first report of TS associated with 22q11DupS. The incidence of TS in the general population is sufficiently high that this association could be incidental. However, a 22q11.2 


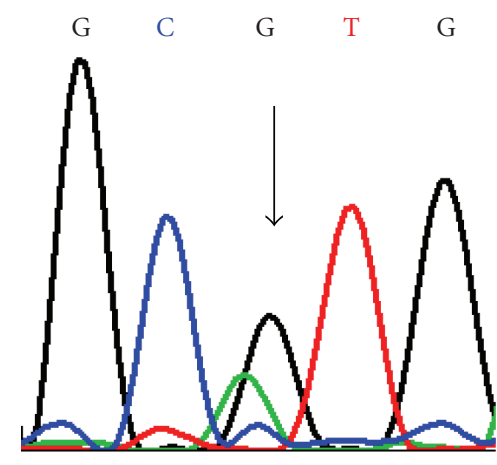

(i)

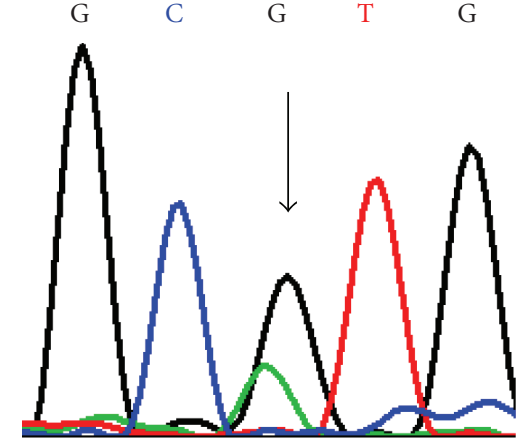

(ii)

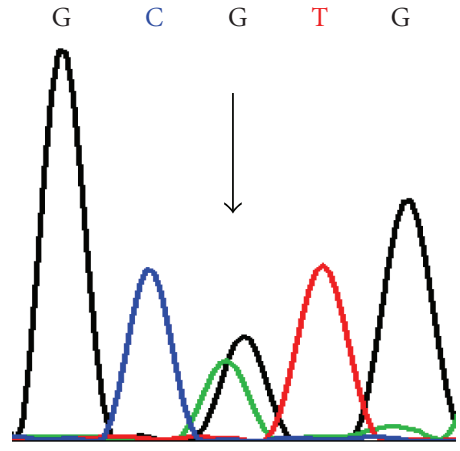

(iii)

(a)

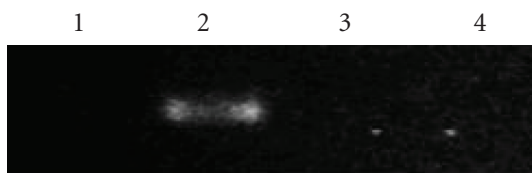

-COMT variant A

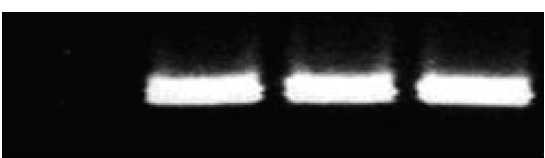

-COMT variant G

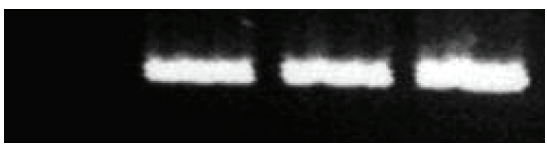

$-\beta$-actin

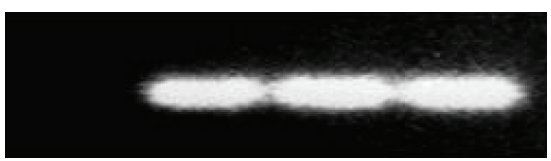

-GAPDH

(b)

FIgURE 2: (a) Nucleotide sequence chromatograms across the COMT G675ㅆ haplotype using (i) patient DNA, (ii) patient cDNA, (iii) mRNA/cDNA from a normal control heterozygote. (b) Expression of the COMT G675A variants. COMT amplicons from semiquantitative RT-PCR were visualized on an agarose gel after 33 cycles using $\beta_{2}$-actin and GAPDH (housekeeping genes) as the quantitation control. Lane 1 - no cDNA control, lane 2-patient, lane $3 \& 4$-normal control individuals that were homozygous for the $675 \underline{G}$ allele.

deletion has been reported in association with tics and TS [7], and as neither parent had a personal or family history of these neuropsychiatric symptoms, our results suggest that the 22q11DupS may confer risk for the TS phenotype.

Hemizygous microdeletion at 22q11.2 (22q11DS) is the most common microdeletion syndrome, usually involving homologous recombination of the same $\sim 3 \mathrm{Mb}$ region duplicated in our patient [13]. Within the 22q11.2 duplication region, candidate genes implicated in central nervous system structure or function include CLTCL1, RTN4R, SNAP29, PRODH, GSCL, UFDIL, ES2, and COMT. The COMT gene (MIM no. 116790) has particular interest because of its role in monoamine degradation and because a common $675 \underline{G}>\underline{A}$ (Val158Met) substitution results in the dysregulation of the dopaminergic system. The $675 \mathrm{~A}$ allele of the COMT haplotype codes for the ${ }^{158} \mathrm{Met}$ variant of this membrane-bound enzyme that is expressed at lower levels in the brain compared to the alternate ${ }^{158} \mathrm{Val}$ variant [14]. A recent investigation of 126 normal healthy Caucasian subjects indicated the COMT low-expression/low-activity $\underline{\mathrm{A}}$ allele forms part of a more expansive COMT gene haplotype "G-A-A" that appears to associate with inefficient prefrontal working-memory response [15]. This same COMT haplotype (G-A-A) also appears to be more prevalent in 22q11DS patients with OCD and ADHD [16].

Both low copy number in 22q11DS and reduced COMT expression due to the presence of a COMT 675 allele [14] have been associated with neuropsychiatric symptoms, including anxiety and Obsessive Compulsive Symptoms [17, 18]. Cerebrospinal fluid neurotransmitter and metabolite levels were not altered in our patient. Although this finding might have had treatment implications, it was not surprising. A recent report showed no alterations in these studies in psychiatric patients with COMT gene polymorphisms [19]. Another study found CSF dopamine changes in Restless Legs Syndrome, but mainly in the presence of more severe symptoms [20]; our patient has milder motor tic symptoms. Finally, it is worth pointing out that studies have questioned 
the role for low COMT activity in psychiatric disorders $[21,22]$. Our patient was heterozygous for the G675스 COMT haplotype with duplication or the $675 \underline{G}$, higher-expressing COMT allele. As such, the 22q11.2 duplication for this patient calls into question a unique role of low COMT activity in the etiopathogenesis of OCD or TS.

Outside the brain, the 22q11DS and 22q11DupS manifest considerable phenotypic overlap, including congenital heart defects, palatal defects and abnormal facies, micrognathia, short stature, dysplastic ears and hearing defects, downslanting palpebral fissures, urogenital anomalies, absent thymus, T cell deficiency, anomalies of the hands and feet, and scoliosis, suggesting diverse effects of both low and high copy numbers [23]. Mouse studies indicate that many of the physical anomalies associated with copy number variation at 22q11.2 are attributable to Tbx1 dosage [24]. Mutations in the GDF6 gene, which is expressed in the developing intervertebral space, are causative for the KF2 class of KFS [25]. $T b x 1$ is likewise expressed in the developing intervertebral space $[25,26]$. The 22q11DS has shown prior association with synostosis in the appendages, scoliosis, and vertebral irregularities [9]; however, this is the first report of vertebral fusion in association with duplication or deletion at 22q11.2. We propose $T b x 1$ as a candidate gene for broad spectrum KFS where it presents in association with Sprengle's shoulder, heart, and palatal and/or urogenital anomalies.

\section{Conclusion}

This study is the first to report an association between TS and 22q11DupS, thereby broadening the link between copy number variation at 22q11.2 and the development of TS and comorbid neurobehavioral disorders including OCB. Further research should delineate the basis for linkage between one or more genes within or near the $22 \mathrm{q} 11.2 \sim 3 \mathrm{Mb}$ critical recombination region and neuropsychiatric disorders.

\section{Acknowledgments}

Data in this case report were presented in part at the 5th International Scientific Symposium on Tourette Syndrome, New York City, New York, USA, June 12-13, 2009. Research support was provided by an Australian Research Council linkage Grant and by National Institute of Neurological Disorders and Stroke R01 NS056276. We gratefully acknowledge this boy and his family for reviewing the manuscript and Matthew W. State, M.D., Ph.D. from the Yale Child Study Center for valuable input.

\section{References}

[1] M. R. Tracy, J. P. Dormans, and K. Kusumi, "Klippel-Feil syndrome: clinical features and current understanding of etiology," Clinical Orthopaedics and Related Research, vol. 424, pp. 183-190, 2004.

[2] H. S. Singer, "Tourette's syndrome: from behaviour to biology," The Lancet Neurology, vol. 4, no. 3, pp. 149-159, 2005.

[3] K. M. Harris, E. M. Mahone, and H. S. Singer, "Nonautistic motor stereotypies: clinical features and longitudinal follow-up," Pediatric Neurology, vol. 38, no. 4, pp. 267-272, 2008.

[4] F. Cardoso and J. Jankovic, "Movement disorders," Neurologic Clinics, vol. 11, no. 3, pp. 625-638, 1993.

[5] C. E. Healy, "Gilles de la Tourette's syndrome (maladie des tics). Successful treatment with haloperidol," American Journal of Diseases of Children, vol. 120, pp. 62-63, 1970.

[6] D. F. Wong, J. R. Brasic, H. S. Singer, et al., "Mechanisms of dopaminergic and serotonergic neurotransmission in Tourette syndrome: clues from an in vivo neurochemistry study with PET," Neuropsychopharmacology, vol. 33, no. 6, pp. 1239-1251, 2008.

[7] M. M. Robertson, B. P. Shelley, S. Dalwai, et al., "A patient with both Gilles de la Tourette's syndrome and chromosome 22q11 deletion syndrome: clue to the genetics of Gilles de la Tourette's syndrome?" Journal of Psychosomatic Research, vol. 61, no. 3, pp. 365-368, 2006.

[8] D. Gothelf, E. Michaelovsky, A. Frisch, et al., "Association of the low-activity COMT 158Met allele with ADHD and OCD in subjects with velocardiofacial syndrome," International Journal of Neuropsychopharmacology, vol. 10, no. 3, pp. 301308, 2007.

[9] A. K. Ryan, J. A. Goodship, D. I. Wilson, et al., "Spectrum of clinical features associated with interstitial chromosome 22q11 deletions: a European collaborative study," Journal of Medical Genetics, vol. 34, no. 10, pp. 798-804, 1997.

[10] M. F. Portnoi, "Microduplication 22q11.2: a new chromosomal syndrome," European Journal of Medical Genetics, vol. 52, no. 2-3, pp. 88-93, 2009.

[11] J. Chen, B. K. Lipska, N. Halim, et al., "Functional analysis of genetic variation in catechol-O-methyltransferase (COMT): effects on mrna, protein, and enzyme activity in postmortem human brain," American Journal of Human Genetics, vol. 75, no. 5, pp. 807-821, 2004.

[12] R. Fukumura, H. Takahashi, T. Saito, et al., "A sensitive transcriptome analysis method that can detect unknown transcripts," Nucleic Acids Research, vol. 31, no. 16, article e94, 2003.

[13] L. Edelmann, R. K. Pandita, E. Spiteri, et al., "A common molecular basis for rearrangement disorders on chromosome 22q11," Human Molecular Genetics, vol. 8, no. 7, pp. 1157$1167,1999$.

[14] J. Chen, B. K. Lipska, N. Halim, et al., "Functional analysis of genetic variation in catechol-O-methyltransferase (COMT): effects on mrna, protein, and enzyme activity in postmortem human brain," American Journal of Human Genetics, vol. 75, no. 5, pp. 807-821, 2004.

[15] A. Meyer-Lindenberg, T. Nichols, J. H. Callicott, et al., "Impact of complex genetic variation in COMT on human brain function," Molecular Psychiatry, vol. 11, no. 9, pp. 867-877, 2006.

[16] E. Michaelovsky, D. Gothelf, M. Korostishevsky, et al., "Association between a common haplotype in the COMT gene region and psychiatric disorders in individuals with 22q11.2DS," International Journal of Neuropsychopharmacology, vol. 11, no. 3, pp. 351-363, 2008.

[17] D. Gothelf, G. Presburger, A. H. Zohar, et al., "Obsessivecompulsive disorder in patients with velocardiofacial (22q11 deletion) syndrome," American Journal of Medical Genetics, Part B, vol. 126B, no. 1, pp. 99-105, 2004.

[18] M. B. Stein, M. D. Fallin, N. J. Schork, et al., "COMT polymorphisms and anxiety-related personality traits," Neuropsychopharmacology, vol. 30, no. 11, pp. 2092-2102, 2005. 
[19] G. Zalsman, Y. Y. Huang, J. M. Harkavy-Friedman, et al., "Relationship of MAO-A promoter (u-VNTR) and COMT (V158M) gene polymorphisms to CSF monoamine metabolites levels in a psychiatric sample of caucasians: a preliminary report," American Journal of Medical Genetics, Part B, vol. 132B, no. 1, pp. 100-103, 2005.

[20] R. P. Allen, J. R. Connor, K. Hyland, et al., "Abnormally increased CSF 3-Ortho-methyldopa (3-OMD) in untreated restless legs syndrome (RLS) patients indicates more severe disease and possibly abnormally increased dopamine synthesis," Sleep Medicine, vol. 10, no. 1, pp. 123-128, 2009.

[21] A. Azzam and C. A. Mathews, "Meta-analysis of the association between the catecholamine-O-methyl-transferase gene and obsessive-compulsive disorder," American Journal of Medical Genetics, Part B, vol. 123B, no. 1, pp. 64-69, 2003.

[22] N. Craddock, M. J. Owen, and M. C. O'Donovan, “The catechol-O-methyl transferase (COMT) gene as a candidate for psychiatric phenotypes: evidence and lessons," Molecular Psychiatry, vol. 11, no. 5, pp. 446-458, 2006.

[23] B. Funke, J. A. Epstein, L. K. Kochilas, et al., "Mice overexpressing genes from the $22 \mathrm{q} 11$ region deleted in velo-cardiofacial syndrome/DiGeorge syndrome have middle and inner ear defects," Human Molecular Genetics, vol. 10, no. 22, pp. 2549-2556, 2001.

[24] S. Merscher, B. Funke, J. A. Epstein, et al., "TBX1 is responsible for cardiovascular defects in velo-cardio-facial/DiGeorge syndrome," Cell, vol. 104, no. 4, pp. 619-629, 2001.

[25] M. Tassabehji, Z. M. Fang, E. N. Hilton, et al., "Mutations in GDF6 are associated with vertebral segmentation defects in Klippel-Feil syndrome," Human Mutation, vol. 29, no. 8, pp. 1017-1027, 2008.

[26] C. Zweier, H. Sticht, I. Aydin-Yaylagul, et al., "Human TBX1 missense mutations cause gain of function resulting in the same phenotype as 22q11.2 deletions," American Journal of Human Genetics, vol. 80, no. 3, pp. 510-517, 2007. 


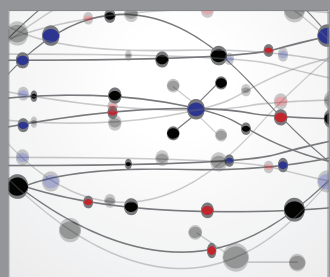

The Scientific World Journal
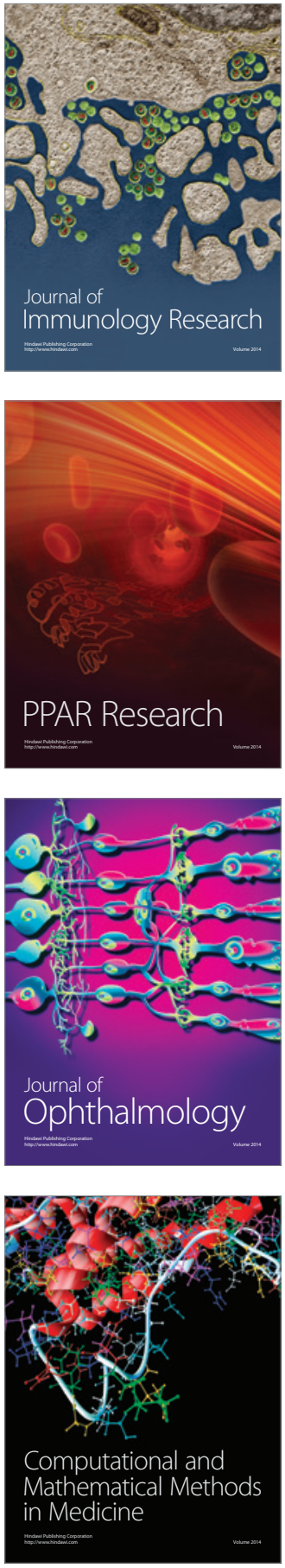

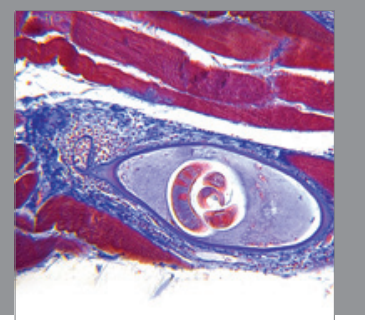

Gastroenterology

Research and Practice
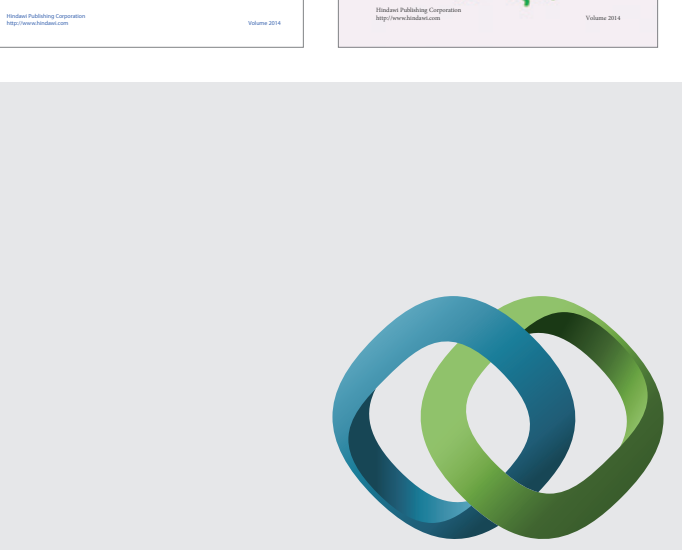

\section{Hindawi}

Submit your manuscripts at

http://www.hindawi.com
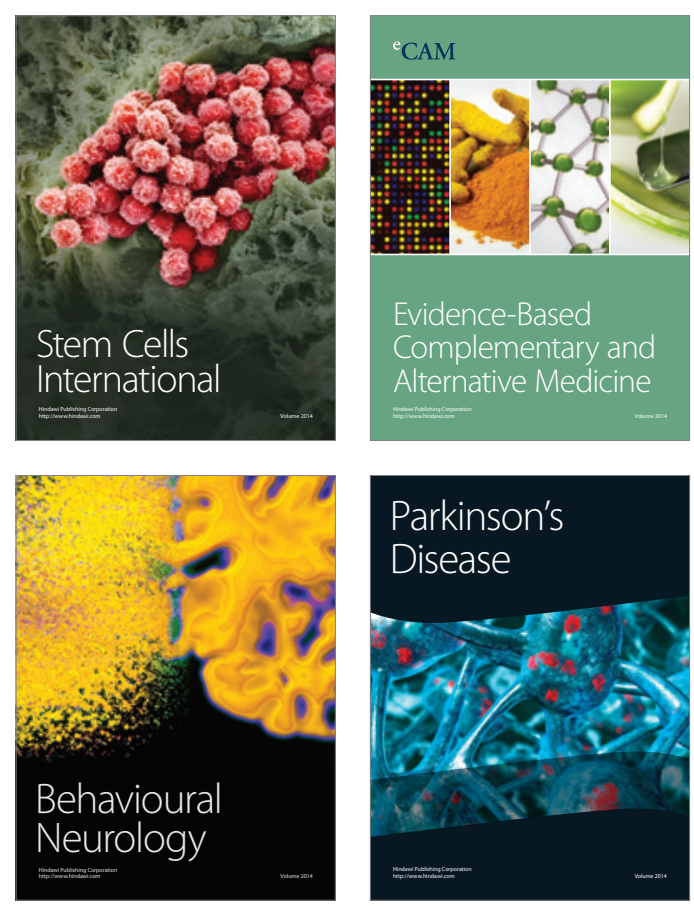

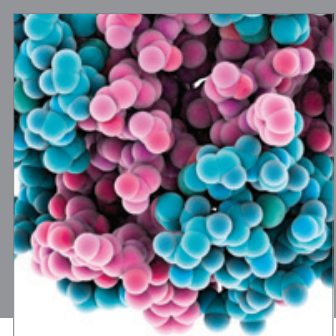

Journal of
Diabetes Research

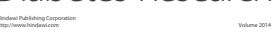

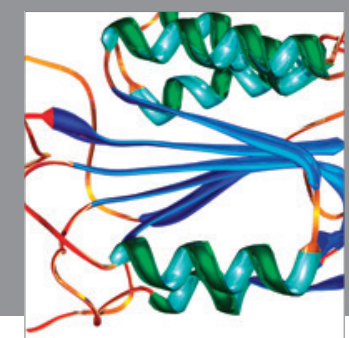

Disease Markers
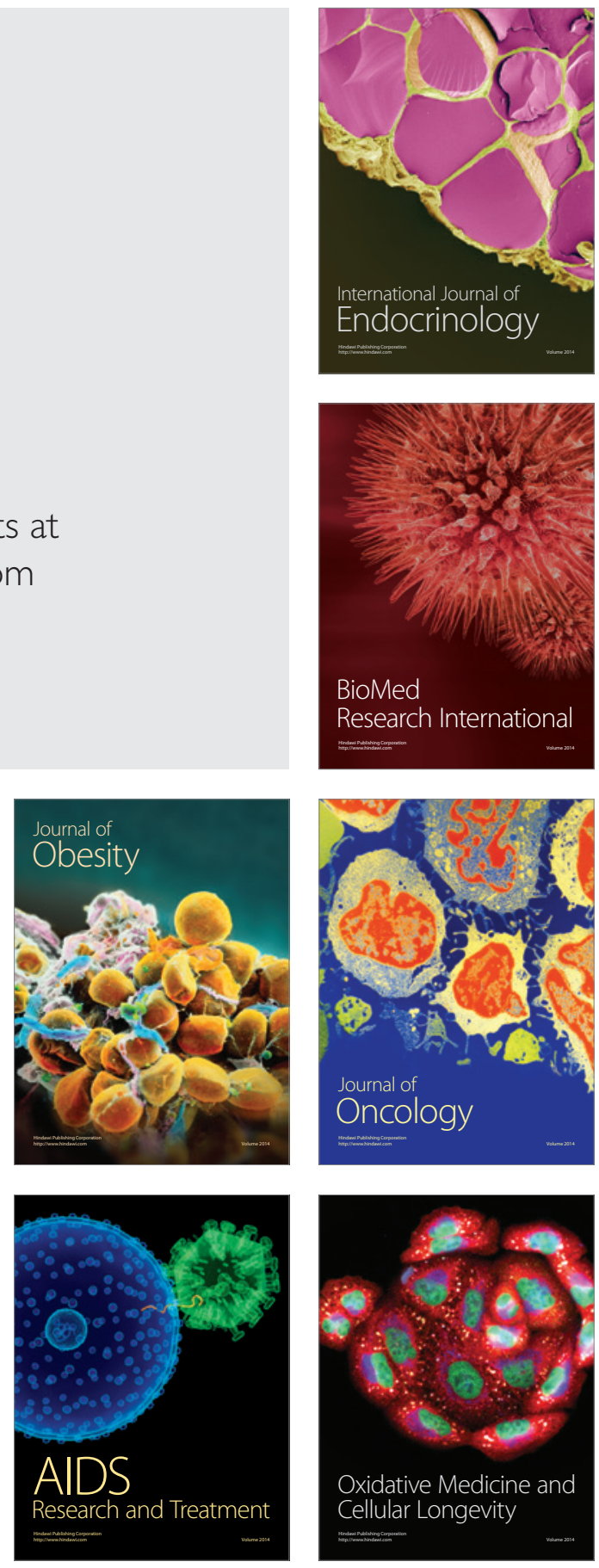